\title{
Identification of spliced mRNA isoforms of retinoid $X$ receptor $(R X R)$ in the Oriental freshwater prawn Macrobrachium nipponense
}

\author{
Z. Li ${ }^{1}$, W.Q. Wang ${ }^{1}$, E.F. Zhang ${ }^{1}$ and G.F. Qiu ${ }^{1,2}$
}

${ }^{1}$ Key Laboratory of Exploration and Utilization of Aquatic Genetic Resources Certificated by Ministry of Education, College of Fisheries and Life Science, Shanghai Ocean University, Shanghai, China

${ }^{2}$ E-Institute of Shanghai Universities, Shanghai Ocean University, Shanghai, China

Corresponding author: G.F. Qiu

E-mail: gfqiu@shou.edu.cn

Genet. Mol. Res. 13 (2): 3914-3926 (2014)

Received February 27, 2013

Accepted July 28, 2013

Published May 23, 2014

DOI http://dx.doi.org/10.4238/2014.May.23.2

\begin{abstract}
Retinoid X receptors (RXR) are members of the nuclear receptor family that are conserved from invertebrates to vertebrates, and they play an essential role in regulating reproductive maturation, molting, and embryo development. In this study, five RXR isoforms, named RXRL2 (L, long form), RXRL3, RXRS1 (S, short form), RXRS2, and RXRS3, containing six domains from A to F, were cloned from the prawn Macrobrachium nipponense using 5'- and 3'- rapid amplification of cDNA ends. Differences among their structures were observed not only in the $\mathrm{D}$ and $\mathrm{E}$ domains but also in the $\mathrm{A} / \mathrm{B}$ domain, which were previously found in insects but not in crustaceans. This is the first report to show that differences occur in the A/B domain of RXR in crustaceans. RXR expressions were also examined in various tissues including the ovary, testis, muscle, hepatopancreas, heart, gill, stomach, intestine, and cuticle. Expression pattern investigations indicated that the five isoforms were differentially expressed. RXRS3
\end{abstract}


was only detected in the ovary, and the other RXRs were abundant in the ovary and testis. These data suggested that RXR mediates a series of processes related to reproduction.

Key words: Retinoid X receptor; Macrobrachium nipponense; Cloning; Distribution; Isoforms

\section{INTRODUCTION}

Retinoid X receptors (RXRs) are the most highly conserved members of the nuclear receptors superfamily. RXR was first isolated from the cDNA library of human liver and kidney cells using human retinoic acid receptor (RAR) DNA in combination with regional cDNA (Mangelsdorf et al., 1990). RXRs have been reported in many species from invertebrates to vertebrates (Wiens et al., 2003). In vertebrates, RXR can bind with RAR to form a heterodimer. Upon activation by 9-cis retinoid acid (RA), the RXR/RAR heterodimer regulates the expression of genes involved in many distinct stages during early embryogenesis, organogenesis, and the transduction of the RA signal during prenatal development (Mark and Chambon, 2003; Mark et al., 2006). RXRs can also form homodimers or heterodimers with the liver X receptor (LXR), the thyroid hormone receptor (TR), the pregnane X receptor (PXR), and other nuclear receptors (Waxman, 1999). Hence, RXR mediates various regulatory activities that are important to the normal physiology of vertebrates. In invertebrates, especially crustaceans and insects, RXR can heterodimerize with the ecdysone receptor (EcR). The heterodimer EcR/ RXR induces the expression of early ecdysone responsive genes, such as transcription factors E74, E75, and Broad Complex, which regulate further downstream cascade reactions by binding to hormone responsive elements (Li and White, 2003; Riddiford et al., 2003).

RXR shares the common six domains (A-F) with other nuclear receptors (Renaud and Moras, 2000; Aranda and Pascual, 2001; Bonneton et al., 2003). The A/B domain at the $\mathrm{N}$-terminal contains a transcriptional activation function (AF-1) sequence in some members, and its length and amino acids vary among species (Aranda and Pascual, 2001; Cherbas et al., 2003). The $\mathrm{C}$ region is highly conserved and acts as a DNA binding domain (DBD), including two zinc-finger motifs (Devarakonda et al., 2003). The E region acts as a ligand-binding domain (LBD), harboring transactivation (AF-2) motifs and a hydrophobic pocket for ligands, and it is associated with heterodimerization (Wang et al., 2000; Hu et al., 2003). The D domain is a flexible hinge region linking the $\mathrm{C}$ and $\mathrm{E}$ domains. The $\mathrm{F}$ region is at the $\mathrm{C}$-terminal, which varies in length and sequence and may be entirely absent in some species.

Due to splice variants in post-transcription, RXR has multiple isoforms in various organisms. In vertebrates, three isoforms $\operatorname{RXR} \alpha, \operatorname{RXR} \beta$, and $\mathrm{RXR} \gamma$, were identified (Tallafuss et al., 2006; He et al., 2009). The main differences among the three isoforms occur in the Nterminal sequences, and their tissue distributions are different. In Sebastiscus marmoratus, three isoforms were isolated with differences in the H1-H3, H7, and $\mathrm{H} 12$ helices. According to the expression of the three isoforms in embryo development stages, it was deduced that $\mathrm{RXR} \alpha$ might be involved in the development of the neural crest, anterior hindbrain, tailbud, and in the formation of the fin and pharynx, whereas RXR $\beta$ likely plays important roles in early development, and that RXR $\gamma$ appears to play a role in the development and function of the nervous system and brain (He et al., 2009). In mature adult rodents, RXR $\alpha$ was found to 
be abundant in the liver, kidney, spleen, and cuticle, while RXR $\beta$ was expressed in almost all tissues, and predominantly in the central nervous system. In contrast, the expression of RXR $\gamma$ was found to be limited to muscle and the peripheral nervous system (Chung and Cooney, 2003). In invertebrates, especially crustaceans and insects, the numbers of RXR isoforms also varies. For instance, in the land crab (Gecarcinus lateralis), nine splicing isoforms were produced by three splicing sites, with one site in the D domain and two in the E domain. More isoforms were produced in the gonad than in skeletal muscles, which indicate a greater complexity of the RXR isoforms in gonads (Kim et al., 2005). In the fiddler crab (Uca pugilator), four RXR isoforms were produced by two splicing sites, with one site in the D domain and the other in the E domain (Hopkins et al., 2008). In the cockroach (Bg, Blattella germanica), two RXR isoforms were isolated, named Bg-RXRL (long form) and Bg-RXRS (short form), with a 23-amino acid insertion/deletion in the H1-H3 helices of the LBD. During embryogenesis, Bg-RXRL was detected at the early stages, while Bg-RXRS was highly expressed in the middle and late stages. This expression pattern strongly suggested that RXR was related to processes in the embryogenesis and the Bg-RXRL might be of maternal origin (Maestro et al., 2005). Many insect RXR isoforms show differences in the $\mathrm{A} / \mathrm{B}$ domain, except for $B$. germanica and migratory locust.

To date, RXR full sequences and their isoforms' expression patterns and functions have been isolated and detected in both land crab and fiddler crab independently, which both belong to the decapod crustacean group (Durica et al., 2002; Kim et al., 2005). The Oriental freshwater prawn, Macrobrachium nipponense, which also belongs to the Palaemonidae family of decapod crustaceans, is an economically and nutritionally important species. In order to gain a better understanding of the expression and function of RXR in crustaceans, in this study, we cloned and characterized the MnRXRs with respect to sequence comparison, 3Dmodel, phylogeny, and tissue expression. Together, these results have led to a hypothesis that crustacean RXRs present more isoforms than those of insects and vertebrates.

\section{MATERIAL AND METHODS}

\section{Animals and sample collection}

Adult prawns, M. nipponense, larger than $6 \mathrm{~cm}$ in length, were purchased from a market in Shanghai, China. They were sacrificed in order to collect tissue samples such as the ovary, testis, heart, muscle, hepatopancreas, intestine, and gill. All tissues were frozen immediately in liquid nitrogen and stored at $-80^{\circ} \mathrm{C}$ until used.

\section{Cloning of RXR cDNAs}

Total RNA was extracted from the ovaries of prawns using Trizol reagent (Invitrogen, USA) as described in the manufacturer protocol, and treated with RNase-free DNaseI (Invitrogen). RNA was reverse transcribed to cDNA with oligo (dT) primers using RT M-MLV (RNase H-, TaKaRa, Japan). The cDNA was used as a template in subsequent polymerase chain reaction (PCR).

Degenerate primers, RXR-DF and RXR-DR (Table 1), were designed based on the highly conserved $\mathrm{C}$ domain of several homologous proteins in the GenBank database (http:// 
www.ncbi.nlm.nih.gov/genbank/), which were used to amplify a partial sequence encoding the $M$. nipponense homolog of RXR. PCR was performed in $25 \mu \mathrm{L}$ volumes including $1 \mu \mathrm{L}$ cDNA primer, $4 \mu \mathrm{L}$ PCR buffer, $2 \mu \mathrm{L}$ dNTP, $1 \mu \mathrm{L}$ upstream, $1 \mu \mathrm{L}$ downstream, $0.2 \mu \mathrm{L}$ DNA polymerase, and $15.8 \mu \mathrm{L} \mathrm{ddH}_{2} \mathrm{O}$. Samples were run on a PCR machine, Mastercycle Pro $\mathrm{S}$ (Eppendorf, Germany), as follows: 40 cycles of denaturation at $94^{\circ} \mathrm{C}$ for $30 \mathrm{~s}$, annealing at $50^{\circ} \mathrm{C}$ for $30 \mathrm{~s}$, then extension at $72^{\circ} \mathrm{C}$ for $45 \mathrm{~s}$, then post-extension at $72^{\circ} \mathrm{C}$ for $5 \mathrm{~min}$. Amplification products were resolved on $1.2 \%$ agarose gel, excised, recovered using a purification system, and then linked into a pGM-T vector, transformed into competent cells, screened white bacterial colonies, and then directly sequenced.

Rapid amplification of cDNA ends (RACE) was used to obtain full-length sequences. The cloning strategy is shown in Figure 1, and the primers are listed in Table 1. For the 3'-sequence, the 3'-Full RACE Core Set (TaKaRa) was used. The specific primers, 3RXRS1 and 3RXRS2 (Table 1), were designed according to the conserved sequence obtained above. Briefly, first-strand cDNA synthesis reactions were performed according to the 3'-Full RACE Core Set. Nested PCR was conducted with a gene-specific primer, 3RXRS1, and the 3'-RACE Outer Primer as the first-round PCR under the following conditions: 25 cycles $\left(94^{\circ} \mathrm{C}\right.$ for $30 \mathrm{~s}$, $65^{\circ} \mathrm{C}$ for $30 \mathrm{~s}$, and $72^{\circ} \mathrm{C}$ for $1 \mathrm{~min}$ ) and then extension at $72^{\circ} \mathrm{C}$ for $10 \mathrm{~min}$. Next, the secondround PCR was performed with the 3RXRS2 primer and the $3^{\prime}$-RACE Inner Primer under the same conditions. PCR products were cloned and sequenced as described above.

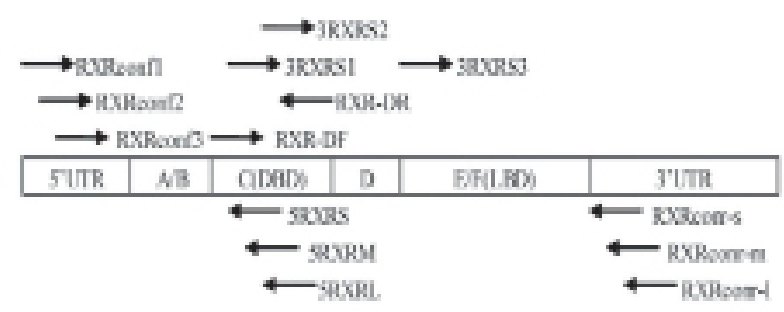

Figure 1. Location of the primers used for cloning of MnRXRs.

The SMART ${ }^{\mathrm{TM}}$ RACE cDNA Amplification Kit (TaKaRa) was used to obtain the 5'end sequences of each cDNA. The downstream specific primers were designed according to the sequences obtained by 3'-RACE, and were named 5RXRS, 5RXRM, and 5RXRL (Table 1). After the first-strand cDNA synthesis, first-round PCR was performed under the following conditions: 5 cycles $\left(94^{\circ} \mathrm{C}\right.$ for $30 \mathrm{~s}, 68^{\circ} \mathrm{C}$ for $30 \mathrm{~s}$, and $72^{\circ} \mathrm{C}$ for $\left.2 \mathrm{~min}\right), 20$ cycles $\left(94^{\circ} \mathrm{C}\right.$ for 30 $\mathrm{s}, 65^{\circ} \mathrm{C}$ for $30 \mathrm{~s}$, and $72^{\circ} \mathrm{C}$ for $3 \mathrm{~min}$ ), and then extension at $72^{\circ} \mathrm{C}$ for $10 \mathrm{~min}$. Second-round PCR was conducted using nested gene-specific primer and a nested universal primer. The PCR conditions were the same as those used for first-round PCR. PCR products were cloned and sequenced as described above.

The sequences obtained from 5'-RACE and 3'-RACE, along with the conserved sequence, was spliced using the Bioedit software. In order to check the integrity of the splicing, specific primers, RXRconf1, RXRconf2, RXRconf3, RXRconr-s, RXRconr-m, and RXRconr1 (Table 1) were designed according to the 5'-untranslated region (UTR) and 3'-UTR separately. PCR products were cloned and sequenced as described above. 


\section{Sequence comparison, phylogenetic analysis and 3D model}

Multiple sequence alignment of the full-length amino acids of Mn-RXRs with known sequences (DQ067280, AF032983, BAF75376.1, NM_011305, P19793, ABF74729, AAF73057) was conducted using the ClustalX software. Phylogenetic analysis of the fulllength RXRs was performed using the neighbor-joining method with 1000 bootstrap repetitions. The consensus phylogenetic tree was assembled using Mega 4.0. The 3D models of Mn-RXRs were created by the Phyre2 web server and labeled with Adobe Photoshop.

\section{Expression of MnRXRs}

Total RNA was extracted from prawn tissues, including the ovary, testis, heart, muscle, hepatopancreas, intestines, stomach, and gill, and treated with RNase-free DNaseI (Invitrogen). Subsequently, cDNAs were synthesized using the Superscript RNase H-reverse transcriptase first strand synthesis system, and quantified based on absorbance at $260 \mathrm{~nm}$. PCR was performed with the gene-specific primers (RXRconr-s and RXRconf1, RXRconr-s and RXRconf2, RXRconr-s and RXRconf3, RXRconr-1 and RXRconf2, and RXRconr-l and RXRconf3) under the following conditions: denaturation at $94^{\circ} \mathrm{C}$ for $3 \mathrm{~min}, 30$ cycles $\left(94^{\circ} \mathrm{C}\right.$ for 30 $\mathrm{s}, 58^{\circ} \mathrm{C}$ for $30 \mathrm{~s}$, and $72^{\circ} \mathrm{C}$ for $30 \mathrm{~s}$ ), and post-extension at $72^{\circ} \mathrm{C}$ for $7 \mathrm{~min}$. The amplification products were then resolved on $1.2 \%$ agarose gel. Meanwhile, $\beta$-actin was used as an internal standard with the same templates.

\section{RESULTS}

\section{Cloning of M. nipponense RXR cDNA}

A 176-bp product was obtained with PCR using degenerated RXR primers based on the partial cDNA sequences encoding the $\mathrm{C}$ domain of known RXRs. The deduced amino acid sequence was $91 \%$ identical to the DBD of Celuca pugilator and Gecarcinus lateralis, $84 \%$ identical to Mus musculus RXR $\alpha$, and $87 \%$ identical with Bombyx mori, indicating that it could be a M. nipponense ortholog of RXRs (Figure 2).

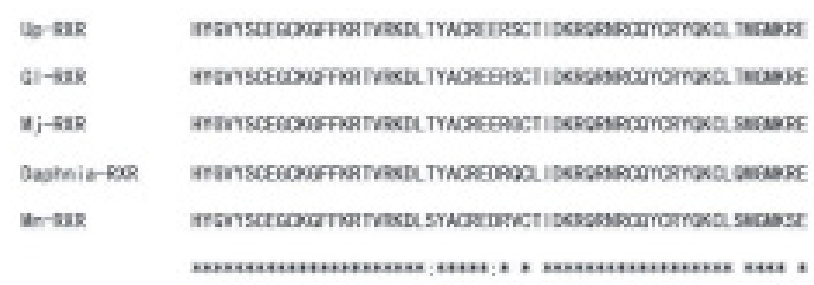

Figure 2. Comparison of deduced amino acid sequences of RXR DNA binding domains.

To obtain the entire cDNAs of RXR, 3'-RACE and 5'-RACE were performed on the basis of the partial sequence. In 3'-RACE, two bands approximately $600 \mathrm{bp}$ and $900 \mathrm{bp}$ in length were obtained. Although RACE-PCR conditions were optimized, three sequences were obtained with lengths of 561, 610, and $885 \mathrm{bp}$, respectively, after the PCR products were 
cloned and sequenced. All three sequences were spliced with the conserved DBD sequence. Subsequently, the amino acid sequences, named Mn-RXRS (short), Mn-RXRM (medium) and Mn-RXRL (long) were confirmed as three isoforms of the RXR 3'-end based on BlastX. In order to obtain the 3'-terminal sequence of Mn-RXRL, the specific primer 3RXRS3 (Table 1) was designed. In 5'-RACE, three sequences, named Mn-RXR1, Mn-RXR2 and Mn-RXR3, were obtained by two bands after cloning and sequencing as for the 3'-RACE. They were also spliced with the conserved sequence, and blasted in the National Center of Biotechnology Information (NCBI), which confirmed that they were three isoforms of the RXR 5'-end. The 3'-and 5'-RACE results revealed nine sequences after splicing, named Mn-RXRS1, Mn-RXRS2, Mn-RXRS3, Mn-RXRM1, Mn-RXRM2, Mn-RXRM3, and Mn-RXRL1, MnRXRL2, and Mn-RXRL3. However, only five isoforms were ultimately obtained in this study. The longest cDNA (Mn-RXRL2) was 1689 bp, which consisted of a 354 bp 5'- UTR, an open reading frame (ORF) of 1011 bp encoding a 337 amino acid sequence, and a 333 bp 3'-UTR. The deduced amino acid sequence of Mn-RXRL2 contained all six domains. The conserved DBD domain included a P-box (FGCKG) and a D-box (CREDR), and the D domain contained the T-box (EAVQEERQ), which is related to the DNA binding domain (Figure 3). Its deduced amino acid was $80 \%$ identical to that of Marsupenaeus japonicus, $84 \%$ identical to Gl-RXRa, $83 \%$ identical to Gl-RXRb, $81 \%$ identical to Gl-RXRc, $76 \%$ identical to Gl-RXRd, $83 \%$ identical to Gl-RXRf, $83 \%$ identical to the C. pugilator RXR, and $68 \%$ identical to the Apis mellifera RXR. The information for the other four isoforms is described in Table 2.

\begin{tabular}{lll}
\multicolumn{2}{c}{ Table 1. Primers used in this study. } & \\
\hline Designation & Primer Sequence & Use \\
\hline RXR-DF & 5'-CACTAYGGSGTSTACAGCTG-3' & Initial amplification, FP \\
RXR-DR & 5'-GCTTCYCKCTTCATSCCCAT-3' & Initial amplification, RP \\
3RXRS1 & 5'-GGAGTATACAGCTGTGAGGGTTGCA-3' & 3'-RACE first-round, FP \\
3RXRS2 & 5'-AGAAGTGTCTATCCATGGGCATG-3' & 3'-RACE nested, FP \\
3RXRS3 & 5'-TGGGTGTTAAAGATGGCATTGTTCT-3' & 3'-RACE nested, FP \\
3' RACE Outer Primer & 5'-TACCGTCGTTCCACTAGTGATTT-3' & 3'-RACE first-round, RP \\
3' RACE Inner Primer & 5'-CGCGGATCCTCCACTAGTGATTTCACTATAGG-3' & 3'-RACE nested, RP \\
5RXRS & 5'-CGTTCGCTGGCGCTCCTCCTGGAC-3' & 5'-RACE, RP \\
5RXRM & 5'-TGTCTGTCAGCTGCCTGGCATGT-3' & 5'-RACE, RP \\
5RXRL & 5'-CCAAGCCCACCTCTATTCCCCCT-3' & 5'-RACE, RP \\
RXRconf1 & 5'-TCGGTAAACGTCTTGGCCTAAG-3' & Splicing verification, FP \\
RXRconf2 & 5'-TCGTGTGACGTTGGCACTTTCG-3' Splicing verification, FP \\
RXRconf3 & 5'-GCCATTGGTCGTTCATCCGTTG-3' & Splicing verification, FP \\
RXRconr-s & 5'-ATGGGGCAATACCAGCTGTG-3' & Splicing verification, RP \\
RXRconr-m & 5'-AGAACCACTCGTGCCGCATC-3' & Splicing verification, RP \\
RXRconr-1 & 5'-ACGATGGATCGTAAACATCC-3' & Splicing verification, RP \\
$\beta$-actinF & 5'-CGACGGTCAGGTCATCACCA-3' & Control of tissue expression, FP \\
-actinR & 5'-ACGTCGCACTTCATGATGGA-3' & Control of tissue expression, RP \\
\hline FP = forward primer; RP = reverse primer.
\end{tabular}

$\mathrm{FP}=$ forward primer; $\mathrm{RP}=$ reverse primer.

Based on comparison of the five isoforms, four variant alternative splicing sites were obtained. A splicing site was located in the amino terminal that generated three variant 5 '-terminal sequences. A second splicing site in the $\mathrm{D}$ domain produced three sequences that differed in the length of the T box, which is a highly conserved sequence adjacent to the DBD. A third splicing site in the H1-H3 helices of the LBD produced two forms. The last one was truncated in the $\mathrm{D}$ domain, and therefore lacked the $\mathrm{E} / \mathrm{F}$ domain. 


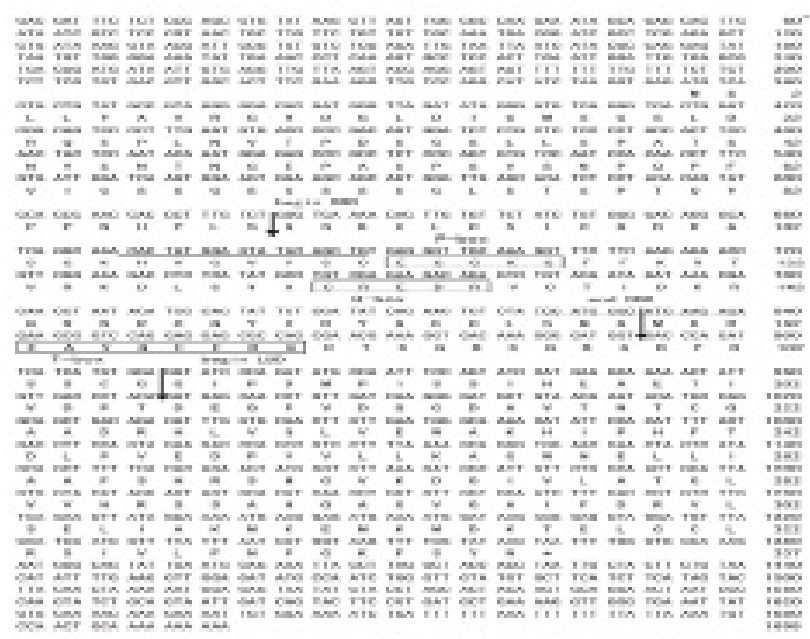

Figure 3. Nucleotide and deduced amino acid sequences of MnRXRL2 cDNAs.

\section{Table 2. Summary of five MnRXR isoforms obtained from RACE.}

\begin{tabular}{llcccrr}
\hline Name & Splice variant & cDNA (bp) & Protein (aa) & Mass (Da) & 5'-UTR (bp) & 3'-UTR (bp) \\
\hline Mn-RXRL2 & N+16/+7/C & 1698 & 337 & 36436 & 354 & 333 \\
Mn-RXRL3 & N0/+12/C & 1659 & 326 & 35292 & 348 & 333 \\
Mn-RXRS1 & N+45/+12/C & 1193 & 277 & 30199 & 263 \\
Mn-RXRS2 & N+16/+7/C & 1346 & 243 & 26397 & 354 & 263 \\
Mn-RXRS3 & N0/+7/C & 1292 & 227 & 24798 & 348
\end{tabular}

$\mathrm{N}=$ for $\mathrm{N}$-terminus; $\mathrm{C}=$ for $\mathrm{C}$-terminus; +16 means that the $\mathrm{A} / \mathrm{B}$ domain has 16 amino acids more than that of RXRL3 and RXRS3; +7 means that the T-box has a short sequence with 7 amino acids; +45 means that the A/B domain has 45 amino acids more than that of RXRL3 and RXRS3; +12 means that the T-box has a short sequence with 12 amino acids. Differences in the C-terminus were complicated and not indicated here.

\section{Sequence comparison, 3D-modeling, and phylogenetic analysis of MnRXR}

To characterize the cloned Mn-RXR, we compared the deduced amino acid sequence of each domain of Mn-RXR with that of some known RXRs from invertebrates (Crustacean and Insecta) and vertebrates (Figure 4). Multiple alignments of the RXR amino acid sequences showed that the N-terminal A/B domain has low homology with other RXRs or ultraspiracles (USPs, an RXR homolog), and the length of the A/B domains are variable. The DBD and LBD domains showed high identity with other RXRs/USPs. The Mn-RXR DBD also contained two zinc fingers for DNA binding. Amino acid sequences in the P-box and D-box of Mn-RXR were identical to other RXRs/USPs, and the T-box was identical to RXRs of fiddler crab, Xenopus, mouse, Daphnia, and Drosophila. The LBD showed divergence between Mn-RXR and other RXRs/USPs, because some of the helices were truncated, especially the H9-H12 sequence. Therefore, we further examined whether these differences influenced tertiary structures and functions of the five isoforms. The 3D models of the five isoforms are shown in Figure 5, and the domains are labeled, especially the helices of the LBD. The structure predictions showed 
that all of the isoforms consisted of a small $\mathrm{C}$ domain linked to a larger LBD domain by a long hinge. Notably, in Mn-RXRL3, Mn-RXRS1 and Mn-RXRS2, the long hinge contained an $\alpha$ helix. The LBD domains appeared to show some similarities and differences. On the one hand, compared with other species, the helices in the LBD of Mn-RXRs were shorter, and for this reason, the helices between $\mathrm{H} 9-\mathrm{H} 12$ were truncated. On the other hand, the number of helices in the LBD domains of Mn-RXRL2 and Mn-RXRL3 were obviously higher than those of the three others, which lack the H6-H8 helices.

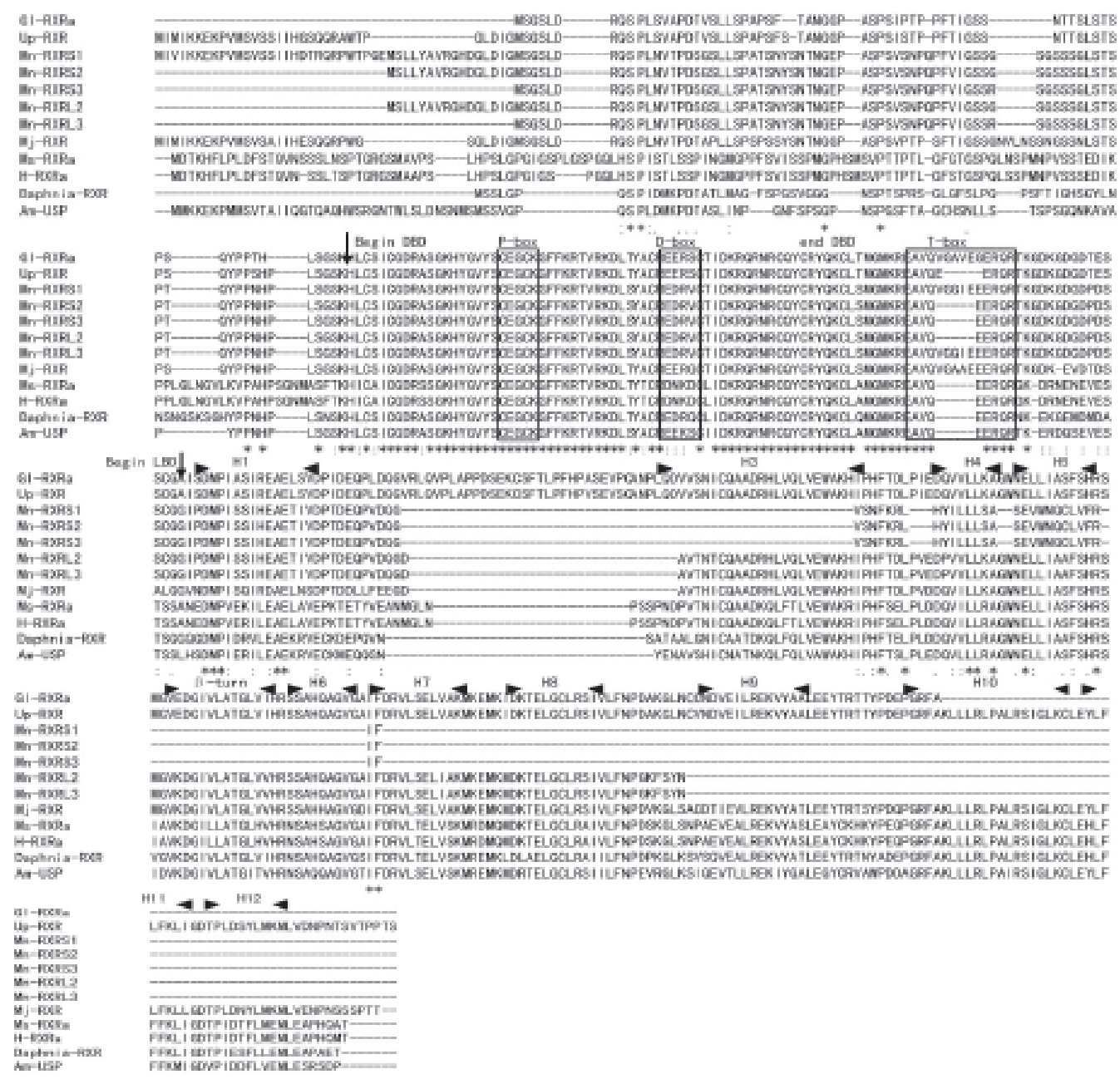

Figure 4. Multiple alignment of amino acid sequences of RXR cDNAs from crustaceans and vertebrates.

Since Mn-RXR sequences showed high homology with the other RXRs, neighborjoining analysis of the full-length RXR amino acid sequences was performed in order to gain perspective on their divergence pattern (Figure 6). RXRs of fiddler crab and land crab were in one branch, and RXRs of the prawns (M. japonicus, M. nipponense) were in the same branch, 
and then clustered as a crustacean clade with Daphnia RXR. In addition, the crustaceans and insects clustered together to form the arthropod clade, and the next branch was composed of the vertebrates. From a systematic point of view, these results suggested that crustaceans and insects are more closely related than they are to vertebrates.
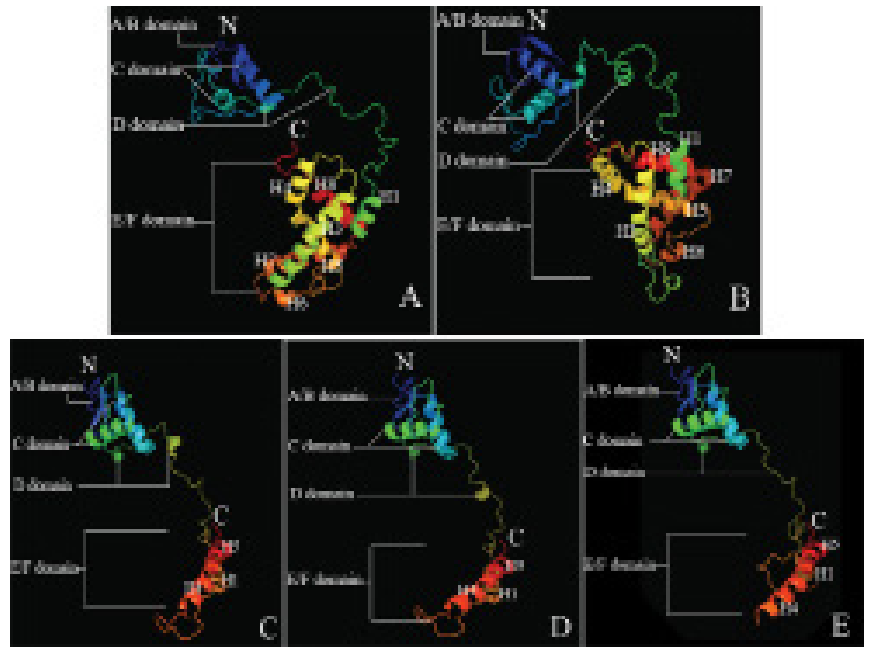

Figure 5. 3-D models of the five isofroms of MnRXR.

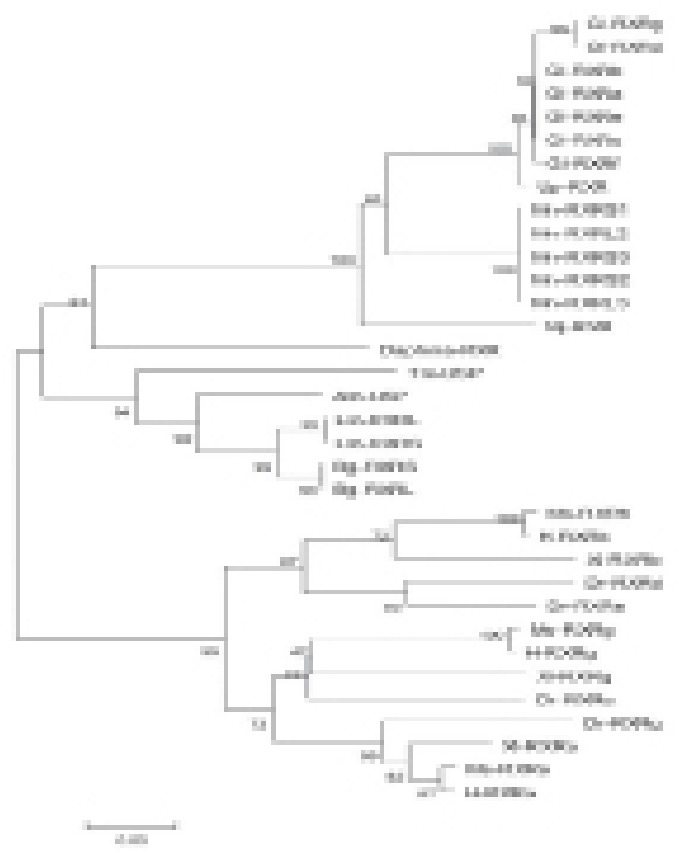

Figure 6. Phylogenetic tree of the full length of RXRs. 


\section{Tissue expression of the prawn RXR}

We performed reverse transcription-PCR (RT-PCR) to examine the tissue-specific expression profile of Mn-RXRs. The five isoforms of Mn-RXR were tested in all of the tested tissues, and $\beta$-actin was used as an internal standard. The same template was used for the five isoforms and $\beta$-actin. The results are showed in Figure 7. Mn-RXRL2 was mainly expressed in the ovary, but was expressed at low levels in the testis, hepatopancreas, and heart. MnRXRL3 expression was the highest in the ovary and testis, but was weak in the muscle and hepatopancreas. Mn-RXRS1 was predominantly expressed in the ovary, and was mildly expressed in the gill and stomach. Mn-RXRS2 was expressed in the ovary and testis at very low levels. In contrast, Mn-RXRS3 was particularly abundant in the ovary.

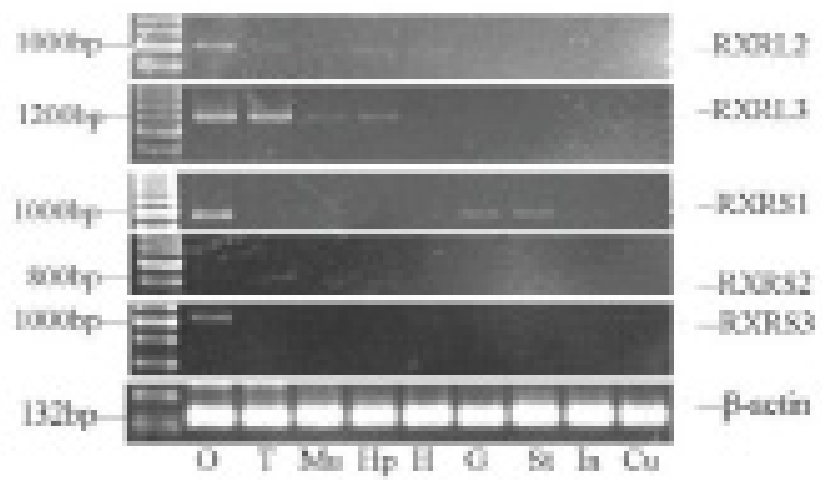

Figure 7. Expression of five isoforms of RXR in the crab tissues.

\section{DISCUSSION}

RXR is a highly conserved member of the steroid nuclear receptor family and plays important roles in several physiological processes of crustaceans, including reproduction and development. Most studies on RXR/USPs have focused on their structure, characterization, and function in vertebrates and invertebrates, whereas there are few reports on the molecular characterization of RXRs in decapod crustaceans. So far, entire cDNA sequences encoding crustacean RXRs have been obtained from fiddler crab C. pugilator (Durica and Hopkins, 1996; Chung et al., 1998), land crab G. lateralis (Kim et al., 2005), water flea D. magna (Wang et al., 2007), kuruma prawn M. japonicus (Asazuma et al., 2007), and Chinese shrimp F. chinensis (Priya et al., 2009). In this study, the full-length cDNA sequences of MnRXRs were isolated from M. nipponense.

Most studies have suggested that there are two or more isoforms of RXR, not only in the vertebrates, but also in invertebrates, including insects and crustaceans. Their common splicing sites were found to be mostly distributed in the T-box of the D domain and in the $\mathrm{H} 1-\mathrm{H} 3$ or H7/8 helices of the E domain (Hopkins et al., 2008), while several RXR isoforms were also shown to differ in their A/B domain sequences, especially in the insects (Horigane et al., 2008). In crustaceans, however, there was no evidence to verify that the isoforms differ 
with respect to their $\mathrm{A} / \mathrm{B}$ domains. In the present study, five sequences of RXR isoforms were found to differ not only in the $\mathrm{D}$ and $\mathrm{E}$ domains, but also in the $\mathrm{A} / \mathrm{B}$ domain. The differences among the five isoforms were as follows. First, the A/B domain has three variant sequences, $\mathrm{A} / \mathrm{B}(+16), \mathrm{A} / \mathrm{B}(+45)$, and $\mathrm{A} / \mathrm{B}(-16)$. Second, there were two variant sequences in the T-box of the D domain, $\mathrm{T}(+12)$ and $\mathrm{T}(+7)$. Third, differences of the LBD were complicated and were distributed across the entire helix (Table 2, Figure 5). Therefore, the present study is the first to demonstrate differences in the A/B domain of crustacean RXRs.

In comparison to other RXRs, the lengths of the LBD of Mn-RXRs obtained in this study were shorter, because H9-H12 helices in the E domain were absent in the Mn-RXRs (Figure 5). Compared with the Up-RXR and Gl-RXR, the helices between H1-H3 of other RXRs were lacking the 33 amino acid (aa) insertion (Figure 3). In the fiddler crab U. pugilator, the results of GST-pull down experiments showed that UpEcR interacted only with UpRXR variants containing the 33 aa insertion, and not with those lacking the 33 aa insertion (Wu et al., 2004). The helices H9-H12 were also obviously truncated in all of the MnRXR isoforms. In Drosophila USP, H12 in the E domain is crucial for DNA binding, efficient ecdysteroid binding by EcR, and transcriptional activation (Przibilla et al., 2004). In crustaceans and insects, RXR can heterodimerize with EcR. The heterodimer EcR-RXR/USP binds to sequence-specific DNA elements and induces the expression of a number of primary response genes, such as transcription factors E74, E75, and Broad Complex (Riddiford et al., 2003). Therefore, it was deduced that the function of these isoforms without the H9-12 region was affected. Moreover, the 3D structures were shown to be significantly different, especially in the LBD domain, which might affect their ligand binding functions.

In addition, other isoforms exist in M. nipponense, which were not obtained in this study. RXRs display a wider variety of isoforms in crustaceans than in insects and vertebrates. There were only two or three RXR/USP isoforms identified in an insect species (Kapitskaya et al., 1996; Jindra et al., 1997), and only three RXR isoforms have been shown to occur in vertebrates, including mice and zebrafish. However, there were potentially four (Up-RXR) or nine (Gl-RXR) alternatively spliced sequences generated at three splicing sites. In the prawn $M$. nipponense, there were five or more isoforms that were generated at four splicing sites. Consequently, multiple isoforms of RXR may represent a characteristic specific to crustacean.

Monitoring the expression of MnRXRs demonstrated that crustacean RXR isoforms showed different expression patterns from that of vertebrates and insects. In S. marmoratus, three RXR isoforms were expressed in all tissues examined, including the brain, eye, gill, heart, ovary, and testis. RXRa and RXRg were expressed at highest levels in the gill, which was consistent with results of Chinese shrimp Fenneropenaeus chinensis, which might suggest that RXR plays an important role in the structure and/or function of gills. RXRb was particularly abundant in gonads, which was similar to other work on mammals (He et al., 2009). In insects, such as in the penultimate and last larval instars of $B$. germanica, Bg-RXRS mRNA was the predominant form in the fat body and in the prothoracic gland. In the adult female, Bg-RXRS mRNA predominated in the fat body, whereas Bg-RXRL mRNA predominated in the ovary (Hopkins et al., 2008). In the prawn, the five RXR isoforms were all expressed in the ovary, and some of them were expressed in the testis, reinforcing the idea that RXR is involved in several physiological processes, including reproduction. RXRS3 was only detected in the ovary, suggesting that it is possibly very important for ovary development.

In summary, our study obtained five isoforms of RXR from M. nipponense by splicing 
following RACE. Sequence comparison and 3D structures suggested that differences occurred in the A/B domain, which has not previously been shown in crustaceans, and that differences in the LBD might influence ligand-binding functions. Compared with vertebrate and other invertebrate species, our results suggest that multiple RXR isoforms might be a characteristic of crustaceans. The expression of RXRs was dominant in the ovary indicating that RXR likely plays an important role in ovary development.

\section{ACKNOWLEDGMENTS}

Research supported by the National KeyTechnology R\&DProgram(\#2012BAD26B04), Shanghai Universities First-class Disciplines Project of Fisheries and the Marine Biology Program of Shanghai Leading Academic Discipline (\#J50701).

\section{REFERENCES}

Aranda A and Pascual A (2001). Nuclear hormone receptors and gene expression. Physiol. Rev. 81: 1269-1304.

Asazuma H, Nagata S, Kono M and Nagasawa H (2007). Molecular cloning and expression analysis of ecdysone receptor and retinoid X receptor from the kuruma prawn, Marsupenaeus japonicus. Comp Biochem. Physiol. B Biochem. Mol. Biol. 148: 139-150.

Bonneton F, Zelus D, Iwema T, Robinson-Rechavi M, et al. (2003). Rapid divergence of the ecdysone receptor in Diptera and Lepidoptera suggests coevolution between ECR and USP-RXR. Mol. Biol. Evol. 20: 541-553.

Cherbas L, Hu X, Zhimulev I, Belyaeva E, et al. (2003). EcR isoforms in Drosophila: testing tissue-specific requirements by targeted blockade and rescue. Development 130: 271-284.

Chung AC, Durica DS and Hopkins PM (1998). Tissue-specific patterns and steady-state concentrations of ecdysteroid receptor and retinoid-X-receptor mRNA during the molt cycle of the fiddler crab, Uca pugilator. Gen. Comp Endocrinol. 109: 375-389.

Chung AC and Cooney AJ (2003). The varied roles of nuclear receptors during vertebrate embryonic development. Nucl. Recept. Signal. 1: e007.

Devarakonda S, Harp JM, Kim Y, Ozyhar A, et al. (2003). Structure of the heterodimeric ecdysone receptor DNA-binding complex. EMBO J. 22: 5827-5840.

Durica DS and Hopkins PM (1996). Expression of the genes encoding the ecdysteroid and retinoid receptors in regenerating limb tissues from the fiddler crab, Uca pugilator. Gene 171: 237-241.

Durica DS, Wu X, Anilkumar G, Hopkins PM, et al. (2002). Characterization of crab EcR and RXR homologs and expression during limb regeneration and oocyte maturation. Mol. Cell Endocrinol. 189: 59-76.

He C, Wang C, Li B, Xie F, et al. (2009). Tissue-specific and embryonic expression of the retinoid X receptors in Sebastiscus marmoratus. Comp Biochem. Physiol. B Biochem. Mol. Biol. 154: 221-228.

Hopkins PM, Durica D and Washington T (2008). RXR isoforms and endogenous retinoids in the fiddler crab, Uca pugilator. Comp Biochem. Physiol. A Mol. Integr. Physiol. 151: 602-614.

Horigane M, Ogihara K, Nakajima Y and Taylor D (2008). Isolation and expression of the retinoid X receptor from last instar nymphs and adult females of the soft tick Ornithodoros moubata (Acari: Argasidae). Gen. Comp Endocrinol. 156: 298-311.

Hu X, Cherbas L and Cherbas P (2003). Transcription activation by the ecdysone receptor (EcR/USP): identification of activation functions. Mol. Endocrinol. 17: 716-731.

Jindra M, Huang JY, Malone F, Asahina M, et al. (1997). Identification and mRNA developmental profiles of two ultraspiracle isoforms in the epidermis and wings of Manduca sexta. Insect. Mol. Biol. 6: 41-53.

Kapitskaya M, Wang S, Cress DE, Dhadialla TS, et al. (1996). The mosquito ultraspiracle homologue, a partner of ecdysteroid receptor heterodimer: cloning and characterization of isoforms expressed during vitellogenesis. Mol. Cell Endocrinol. 121: 119-132.

Kim HW, Lee SG and Mykles DL (2005). Ecdysteroid-responsive genes, RXR and E75, in the tropical land crab, Gecarcinus lateralis: differential tissue expression of multiple RXR isoforms generated at three alternative splicing sites in the hinge and ligand-binding domains. Mol. Cell Endocrinol. 242: 80-95.

Li TR and White KP (2003). Tissue-specific gene expression and ecdysone-regulated genomic networks in Drosophila. 
Dev. Cell 5: 59-72.

Maestro O, Cruz J, Pascual N, Martin D, et al. (2005). Differential expression of two RXR/ultraspiracle isoforms during the life cycle of the hemimetabolous insect Blattella germanica (Dictyoptera, Blattellidae). Mol. Cell Endocrinol. 238: 27-37.

Mangelsdorf DJ, Ong ES, Dyck JA and Evans RM (1990). Nuclear receptor that identifies a novel retinoic acid response pathway. Nature 345: 224-229.

Mark M and Chambon P (2003). Functions of RARs and RXRs in vivo: Genetic dissection of the retinoid signaling pathway. Pure Appl. Chem. 75: 1709-1732.

Mark M, Ghyselinck NB and Chambon P (2006). Function of retinoid nuclear receptors: lessons from genetic and pharmacological dissections of the retinoic acid signaling pathway during mouse embryogenesis. Annu. Rev. Pharmacol. Toxicol. 46: 451-480.

Priya TA, Li F, Zhang J, Wang B, et al. (2009). Molecular characterization and effect of RNA interference of retinoid X receptor (RXR) on E75 and chitinase gene expression in Chinese shrimp Fenneropenaeus chinensis. Comp Biochem. Physiol. B Biochem. Mol. Biol. 153: 121-129.

Przibilla S, Hitchcock WW, Szecsi M, Grebe M, et al. (2004). Functional studies on the ligand-binding domain of Ultraspiracle from Drosophila melanogaster. Biol. Chem. 385: 21-30.

Renaud JP and Moras D (2000). Structural studies on nuclear receptors. Cell Mol. Life Sci. 57: 1748-1769.

Riddiford LM, Hiruma K, Zhou X and Nelson CA (2003). Insights into the molecular basis of the hormonal control of molting and metamorphosis from Manduca sexta and Drosophila melanogaster. Insect Biochem. Mol. Biol. 33: 1327-1338.

Tallafuss A, Hale LA, Yan YL, Dudley L, et al. (2006). Characterization of retinoid-X receptor genes rxra, rxrba, rxrbb and rxrg during zebrafish development. Gene Expr. Patterns. 6: 556-565.

Wang SF, Ayer S, Segraves WA, Williams DR, et al. (2000). Molecular determinants of differential ligand sensitivities of insect ecdysteroid receptors. Mol. Cell Biol. 20: 3870-3879.

Wang YH, Wang G and LeBlanc GA (2007). Cloning and characterization of the retinoid X receptor from a primitive crustacean Daphnia magna. Gen. Comp Endocrinol. 150: 309-318.

Waxman DJ (1999). P450 gene induction by structurally diverse xenochemicals: central role of nuclear receptors CAR, PXR, and PPAR. Arch. Biochem. Biophys. 369: 11-23.

Wiens M, Batel R, Korzhev M and Muller WE (2003). Retinoid X receptor and retinoic acid response in the marine sponge Suberites domuncula. J. Exp. Biol. 206: 3261-3271.

Wu X, Hopkins PM, Palli SR and Durica DS (2004). Crustacean retinoid-X receptor isoforms: distinctive DNA binding and receptor-receptor interaction with a cognate ecdysteroid receptor. Mol. Cell Endocrinol. 218: 21-38. 